\title{
PSMA-targeted PET Imaging Agent Fluorine F 18 CTT-1057
}

National Cancer Institute

\section{Source}

National Cancer Institute. PSMA-targeted PET Imaging Agent Fluorine F 18 CT T-1057.

NCI Thesaurus. Code C132028.

A radioconjug ate composed of the phosphoramidate agent CT T1057, a human prostate specific membrane antigen (PSMA) inhibitor, and labeled with the radioisotope fluorine $F$ 18 , with potential use as a tracer for PSMA-expressing tumors during positron emission tomography (PET). Upon intravenous administration of PSMA-targeted PET imaging agent fluorine F 18 CT T-1057, the CTT-1057 moiety targ ets and irreversibly binds to the extracellular domain of PSMA-expressing tumor cells. Upon rapid internalization, and following PET imaging, PSMA-expressing tumor cells can be detected. PSMA, a tumorassociated antigen (TAA) and type II transmembrane protein, is expressed on the membrane of prostatic epithelial cells and overexpressed on prostate tumor cells. 